\title{
Frédéric Dumas, Mark Twain : Tourisme et Vanité
}

\section{Ella Mingazova}

\section{OpenEdition}

Journals

Édition électronique

URL : http://journals.openedition.org/lectures/19796

ISSN : 2116-5289

Éditeur

Centre Max Weber

Ce document vous est offert par Université de Liège

\section{LIĖGE \\ université}

\section{Référence électronique}

Ella Mingazova, «Frédéric Dumas, Mark Twain : Tourisme et Vanité », Lectures [En ligne], Les comptes rendus, 2016, mis en ligne le 11 janvier 2016, consulté le 17 janvier 2018. URL : http:// journals.openedition.org/lectures/19796

Ce document a été généré automatiquement le 17 janvier 2018.

(c) Lectures - Toute reproduction interdite sans autorisation explicite de la rédaction / Any replication is submitted to the authorization of the editors 


\title{
Frédéric Dumas, Mark Twain : Tourisme et Vanité
}

\author{
Ella Mingazova
}

Spécialiste en littérature américaine, Frédéric Dumas offre une analyse textuelle de quatre livres de voyage de Mark Twain : À la dure, Le Voyage des innocents, Ascensions en télescope et Le Tour du monde d'un humoriste. L'auteur envisage ces quatre récits de voyage comme un ensemble cohérent, unifié par un narrateur commun qu'il nomme «Twain ». En effet, en contre-pied à l'approche biographique de ces ouvrages généralement préférée par la critique, Dumas parle de «Twain » en utilisant les guillemets pour souligner la différence entre le héros métadiégétique ${ }^{1}$ fictif et l'auteur empirique Mark Twain, pseudonyme utilisé par Samuel L. Clemens. En d'autres termes, «Twain» n'est pas le voyageur Twain lui-même mais une persona ignorante et comique fabulée par l'écrivain. Outre cette situation narrative commune aux quatre récits de voyage, Dumas décèle également trois thèmes qui s'entrecroisent: le tourisme, l'intérêt de "Twain» pour l'esthétique et la "Vanité ", en tant que genre pictural du XVII e siècle évoquant la nature éphémère de la vie humaine. Les récits de voyage analysés se situent au XIX ${ }^{e}$ siècle, au tout début du développement du tourisme de masse. Alors que la mauvaise réputation du tourisme, décrié, lamenté et critiqué jusqu'à nos jours² ${ }^{2}$ est aussi vieille que le phénomène lui-même ${ }^{3}$, "Twain » se présente comme un touriste assumé qui voyage pour son plaisir. Dans la première partie de l'ouvrage, Frédéric Dumas démontre comment les récits de ce touriste-dilettante parodient les récits des écrivains-voyageurs, pour qui le voyage doit être semé de difficultés et doit devenir un véritable travail, mot qui est d'ailleurs à l'origine de travel en anglais. Ainsi, alors «qu'en 1878 Stevenson parcourt la campagne française en citant Montaigne et en travaillant son Voyage avec un âne dans les Cévennes, “Twain" visite les Alpes à la mode en se référant constamment à l'œuvre de Baedeker et consort » (p. 67). Cependant, les récits ne font pas l'apologie du tourisme et constituent des versions parodiques des guides touristiques auxquels ils se réfèrent. "Twain» présente le tourisme comme une imposture à travers le motif récurrent de la supercherie. 
2 Pour Dumas, la supercherie constitue un des motifs centraux dans les récits de voyage de «Twain ». Cette supercherie se manifeste tout d'abord par le caractère théâtral de toute activité touristique, où les costumes sont essentiels à l'identification des rôles joués par chacun. Ainsi, les autochtones déguisés jouent «le rôle de l'indigène fantasmé par les touristes, alors que "Twain" affublé comme un touriste modèle, prétend être un aventurier » (p. 95). Les arnaques, essentiellement financières, dont le touriste peut faire l'objet apparaissent comme une autre manifestation de la supercherie. La création de mythes, essentielle dans les discours touristiques, est examinée par Dumas, comme un autre aspect de la supercherie dénoncée par « Twain ». À travers la création de légendes à leur sujet, des lieux, religieux notamment, deviennent des attractions qui suscitent l'engouement des visiteurs. Enfin, la supercherie en tant que discours est également présente dans la narration des récits de voyage eux-mêmes. Fortement influencés par la tradition orale, les récits de "Twain » sont emprunts d'exagérations. Le narrateur se déclare comme simple intermédiaire qui relate des récits entendus, provenant de sources qui restent anonymes. Au-delà d'une simple critique du tourisme, la supercherie soulève donc la question de la médiatisation du réel, plus précisément du décalage entre représentation et réel.

3 Pour Frédéric Dumas, cette problématique de la représentation du réel s'inscrit dans un questionnement plus large de «Twain » au sujet de l'esthétique. L'auteur suggère que la réflexion portant sur la représentation iconique occupe une place importante dans les récits. Les arguments les plus originaux à ce sujet sont développés dans le chapitre IX. Dumas s'y concentre sur le rapport de "Twain » à l'image ainsi que sur les illustrations humoristiques qui renforcent la nature parodique des récits analysés. Même si « Twain » se présente en tant que voyageur anti-intellectuel qui revendique son ignorance et son manque de culture, Frédéric Dumas démontre que ses réflexions au sujet de l'art constituent bel et bien un questionnement sérieux où « il interroge les fondements de l'appréhension du beau » (p. 169). Dès lors, Dumas analyse les récits de voyage non pas comme une simple parodie des guides touristiques mais bien comme une réflexion complexe et ironique sur le genre pictural, la Vanité en particulier.

Intitulé "Le texte-vanité », le chapitre X avance l'argument principal de l'ouvrage et développe l'idée de filiation des récits de «Twain» avec la tradition de la Vanité picturale. L'auteur y suggère qu'une esthétique de la mort est au centre de l'ensemble des quatre récits de voyage, tant au niveau thématique que rhétorique et stylistique. Emprunte du style gothique, l'œuvre de « Twain » « reflète l'angoisse liée à la fugacité de l'existence et à l'inanité de toute production humaine» (p. 238). "Twain » remet en question la nature même de ses voyages et de ses récits, jugés en fin de compte futiles. Il reproduit la juxtaposition d'objets des Vanités et observe, par exemple, l'accumulation machinale d'objets matériels des touristes-consommateurs, de souvenirs en tout genre et notamment des memento mori tels que des ossements trouvés dans le désert, en mettant en évidence leur inutilité au vu de la condition humaine de mortalité. Le narrateur accumule, lui aussi, des citations et des anecdotes au cours de ses voyages pour construire des récits où les digressions abondent. Frédéric Dumas montre que la réflexion autour de la mort est à la fois liée à l'intérêt de «Twain » pour l'esthétique et à sa réflexion sur le phénomène de tourisme naissant dans son rapport au consumérisme.

Dans cet ouvrage volumineux, Frédéric Dumas fait preuve d'une recherche fouillée, notamment nourrie par les archives Mark Twain de l'Université de Berkeley. Dumas défend le caractère innovant des quatre récits de voyage de Mark Twain et décrit un 
narrateur visionnaire qui, derrière des apparences simplistes et un ton léger, émet des opinions très sophistiquées sur des sujets tels que la mort, le sacré, l'appréhension du beau et le consumérisme, pour construire simultanément un récit de voyage moderne jonché de digressions et une œuvre emprunte de Vanité.

\section{NOTES}

1. Le lecteur d'un récit de voyage a tendance à considérer le héros comme métadiégétique, c'està-dire comme étant à la fois l'auteur, le voyageur et le narrateur. Tim Youngs, par exemple, définit le récit de voyage comme " a predominantly factual, first person prose accounts of travels that have been undertaken by the author-narrator " (« un récit à la première personne principalement factuel narrant les voyages qui ont été entrepris par l'auteur-narrateur», nous traduisons). Youngs Tim, The Cambridge Introduction to Travel Writing, Cambridge, Cambridge University Press, 2013, p. 3. Voir aussi Borm Jan, «Defining Travel: On the Travel Book, Travel Writing and Terminology. », in Glenn Hooper and Tim Youngs (dir.), Perspectives on Travel Writing, Aldershot, Ashgate, 2004, p. 17.

2. Entre autres par Daniel J. Boorstin et Paul Fussell. Voir Boorstin Daniel, The Image: A Guide to Pseudo-Events in America, New York, Vintage Books, 1992, recensé par Sophie Maunier pour Lectures dans sa traduction française : http://lectures.revues.org/9014. Voir également Fussell Paul, Abroad: British Literary Travelling Between the War, Oxford, Oxford University Press, 1980.

3. Voir Buzard James, The Beaten Track: European Tourism, Literature, and the Ways to Culture, 1800-1918, Oxford/New York, Oxford University Press, 1993.

\section{AUTHOR}

\section{ELLA MINGAZOVA}

Diplômée de master en langues et littératures modernes de l'Université catholique de Louvain. 